\title{
Correction to: Is there a correlation between HER2 gene amplification level and response to neoadjuvant treatment with trastuzumab and chemotherapy in HER2-positive breast cancer?
}

\author{
Silvia Antolín ${ }^{1}$ (1) - Lucía García-Caballero ${ }^{2}$ (1) - Cristina Reboredo ${ }^{1}$ - Aurea Molina ${ }^{1}$. Joaquín Mosquera ${ }^{1}$. \\ Ángel Vázquez-Boquete ${ }^{3} \cdot$ Rosalía Gallego $^{2} \cdot$ Mari Paz Santiago $^{4} \cdot$ Ángel Concha $^{4} \cdot$ Eva Pérez $^{1}$ (1) $\cdot$ Lourdes Calvo $^{1}$ (1) . \\ Tomás García-Caballero ${ }^{2,3}$ (1)
}

Published online: 12 June 2021

○) Springer-Verlag GmbH Germany, part of Springer Nature 2021

\section{Correction to: Virchows Archiv \\ https://doi.org/10.1007/s00428-021-03104-7}

In the original published version of this article, the affiliations of the co-authors Lucía García-Caballero and Ángel Vázquez-Boquete were incorrectly presented. Lucía GarcíaCaballero affiliation should be "Department of Morphological Sciences, School of Medicine and Dentistry, Santiago de Compostela University, Santiago de Compostela, Spain" and the affiliation of Ángel Vázquez-Boquete should be "Department of Pathology, Santiago de Compostela University Hospital Complex, Santiago de Compostela, Spain.

The original article has been corrected.

Publisher's note Springer Nature remains neutral with regard to jurisdictional claims in published maps and institutional affiliations.

The online version of the original article can be found at https:// doi.org/10.1007/s00428-021-03104-7

Lucía García-Caballero

lucia.garcia.caballero@usc.es

1 Department of Medical Oncology, A Coruña University

Hospital Complex, A Coruña, Spain

2 Department of Morphological Sciences, School of Medicine and Dentistry, Santiago de Compostela University,

Santiago de Compostela, Spain

3 Department of Pathology, Santiago de Compostela University Hospital Complex, Santiago de Compostela, Spain

4 Department of Pathology, A Coruña University Hospital Complex, A Coruña, Spain 